\title{
Toxicity of volatile organic compounds (VOCs) mixtures using human derived cells
}

\author{
C. Khalil \& J. Nasir \\ School of Safety Sciences, \\ Chemical Safety and Applied Toxicology Laboratories, UNSW, Australia
}

\begin{abstract}
Assessing the effects of contaminants is an issue of high priority for governmental safety health and environmental agencies around the world. The general conservative consensus is that chemicals in mixtures interact by concentration addition. However, previous studies also report that concentration addition of mixture components does not always reflect the overall toxicity of a mixture. Volatile organic compounds (VOCs) such as Benzene, Toluene, Xylene and Formaldehyde (BTXF) belong to the air pollutants found in urban and indoor environments. They could trigger acute and chronic adverse health effects like allergy, respiratory and cardiovascular diseases. The volatile nature of these compounds poses additional problems in assessing individual volatile chemical toxicity let alone mixtures of these chemicals. Our research aims at establishing the true toxic effects of VOC exposure in vitro using a static direct exposure glass-chamber method. This was achieved by assessing and comparing individual and interactive effects of VOCs in exposed human epithelial lung (A549) and liver cells (HepG2) using the MTS cytotoxicity assay to assess cell viability upon VOC insult. The study results clearly indicated the limitation of the concentration addition method used in assessing volatile mixtures cytotoxicity and the need to develop new techniques for rapid and accurate mixture toxicity determination. The study may have implications for regulatory risk assessment of environmental volatile organic chemicals.

Keywords: static method, MTS, cytotoxicity, lung cells, liver cells, VOCs.
\end{abstract}

\section{Introduction}

Human environmental chemical exposures are characterised by exposures to direct multiple chemical combinations or sequential exposure to individual or 
different chemicals at low concentrations [1]. These exposures are unavoidable because chemicals represent an integral part of our life and play an important role in promoting human lifestyle and wellbeing. However, data on chemical mixtures is sparse due to the focus of traditional toxicology on individual chemicals and their toxicities, but interest in this emerging area of mixture toxicity has been building in recent years [2].

The main objective of this research is to present findings relevant to assessment of toxicological human health risks as a result of exposure to volatile mixtures. This is achieved through toxicity determination of individual chemical components in a mixture and correlating that to the observed toxicity resulting from the mixture. The selection of biological tests rather than chemical identification as a starting point is based on the Australian and New Zealand Environment and Conservation Council (ANZECC) environmental guidelines for conservation and sustainable development which recognises a hierarchy of evidence based assessment. This assessment ranges from most powerful (biological effects) to least powerful (chemical identification and measurement) evidence [3].

Furthermore, toxicity assessment of chemical mixtures is a challenging task and requires understanding interactions and characteristics of the chemicals present [4]. Unfortunately in the environment, it is not always possible to identify all individual chemicals in a mixture and their interactions [5], hence the need for a rapid, repeatable and accurate in vitro screening technique (e.g. static glass-chamber method) to assess overall toxicity of mixtures. Natural systems are complex systems and it is virtually impossible to understand the full mechanism of migration, accumulation, biotransformation and toxicity of volatile chemicals introduced in such open systems, but it is hoped that the results presented here could elucidate some of the toxic potential of mixtures in a controlled environment [5].

This study investigated airborne concentrations of Benzene, Toluene, Xylene and Formaldehyde and their mixtures. The toxicity assessment was undertaken in human derived cells (lung and liver cells) using a colorimetric assay, the MTS assay. The investigation endpoint aimed at producing dose response curves (for individual and chemical mixtures) to establish the nature of the toxicological effects resulting from chemicals mixtures exposure. The cell culture selection was based on potential targets of exposure in humans (mainly lung and liver). The selected cells by virtue of their location, numbers and ease of growth in culture could be used as possible indicators of cellular damage caused by multiple contaminant exposure in vitro [6]. Furthermore, the techniques used are rapid, reproducible and generate accurate individual and mixtures toxicity profiles within hours of conducting a full set of assays.

\section{Materials and methods}

\subsection{Chemicals}

Benzene (CAS No. 71-43-2) was purchased from BDH Chemicals, Australia (Laboratory reagent). Toluene (C6H5CH3), CAS\# 108-88-3, was purchased 
from APS Finechem, Australia (Analytical reagent). Xylene (CAS No. 1330-207) and Formaldehyde (CAS No. 50-00-0) both were purchased from ChemSupply Australia (Laboratory reagents). In vitro assay reagents were purchased from Promega (USA) and Sigma (USA).

\subsection{Human-derived cell cultures}

The main cell cultures used in this research consisted of an epithelial lung carcinoma cell (A549) [ATCC CCL-185] and a Heptacarcinoma human cell line (HepG2) derived from the tissue of a 15 year old Caucasian male [ATCC HB8065]. Cells were sub-cultured as adherent cells in $75 \mathrm{~cm}^{2}$ tissue culture flasks with $0.2 \mu \mathrm{m}$ vented seals (Falcon). The culture media consisted of colour free Dulbeco's modified eagle medium (DMEM): RPMI 1640 (1:1) purchased from Sigma Chemicals, supplemented with 5\% foetal calf serum (Trace Bioscience), 3\% Sigma antibiotics [penicillin (100 U/ml), streptomycin $(0.1$ $\mathrm{mg} / \mathrm{ml}$ ) and L-glutamine (2 mM)]. Cell lines (HepG2 and lung cells (A549) were cultured at $37^{\circ} \mathrm{C}$ at sub-confluence in a humidified incubator set to a mixture of $5 \% \mathrm{CO}_{2} / 95 \%$ air. Cell viability was over $95 \%$ as measured by tryptan blue dye exclusion. Confluent cells in log phase of growth were released from the bottom of the culture flask using Trypsin EDTA (Gibco, USA), and then washed three times with cell culture medium before being seeded on porous membranes $(0.4$ $\mathrm{mm}$ ) on snapwell inserts.

Snapwell insert is a modified transwell culture insert with a $12 \mathrm{~mm}$ diameter providing a growth area of $1.12 \mathrm{~cm}^{2}$ (clear polyster Snapwellt insert, 3801, Corning), supported by a detachable ring that was placed in a six well culture plate. Culture media and 1\% (v/v) HEPES buffer was added to both sides (bottom, $2 \mathrm{ml}$; top, $0.5 \mathrm{ml}$ ) of the membranes. The snapwell inserts in six well plates were incubated at $37^{\circ} \mathrm{C}$ for one hour as an initial equilibrium time to improve cell attachment. Culture media was then removed from the top and replaced with fresh culture media $(0.5 \mathrm{ml})$ containing a cell suspension, $(20-30)$ x $10^{4}$ cells, supplemented with 5\% FCS, $1 \%$ antibiotics and 1\% HEPES buffer. Cell cultures in six well plates were incubated at $37^{\circ} \mathrm{C}$ in a humidified incubator for $24 \mathrm{~h}$. Cell attachment was observed under the light microscope (Leitz Wtzlar, Germany), medium was removed from both sides of the snapwell inserts and membranes washed with Hank's balanced salt solution (HBSS; Gibco, USA) from both sides (top, $0.5 \mathrm{ml}$; bottom, $2.0 \mathrm{ml}$ ). Cells on the membranes were exposed to airborne concentrations of test chemicals on their apical side while being nourished from their basolateral side, using the static exposure technique.

\subsection{Exposure protocol}

Standard test atmospheres were generated using a static methodology as outlined in Bakand et al. [7]. Briefly, a known quantity of volatile liquid was introduced into the glass bottle onto a filter paper. Human derived cells grown on snapwell inserts were detached from their holders and placed into sterile individual glass wells. Each glass well contained $1.2 \mathrm{ml}$ of serum free culture 
media supplemented with 1\% HEPES buffer. Aliquots of test chemicals (ranging from $0,2.5,5.0,10.0,15.0,20.0$, or $30.0 \mathrm{ml}$ ) were introduced to the glass chambers onto the filter paper. Glass chambers were immediately closed, sealed with parafilm and placed on an orbital mixer incubator (50 RPM; Ratek Instruments, Australia) at $37^{\circ} \mathrm{C}$. Each aliquot of volatile liquid was introduced into a single chamber. Human cells were exposed to various airborne concentrations of volatile test chemicals directly at the air/ liquid interface for $1 \mathrm{~h}$. Details on airborne test concentration calculations can be found in [8].

At the end of the exposure time, snapwell inserts were removed and replaced in their holders within six well plates, Culture media supplemented with $1 \%$ HEPES buffer was added to both sides (top, $0.5 \mathrm{ml}$; bottom, $2 \mathrm{ml}$ ) of the membranes. Cells were incubated for $24 \mathrm{~h}$ at $37{ }^{\circ} \mathrm{C}$ in a humidified incubator. At the end of the incubation time, cell viability was investigated using the MTS (Tetrazolium salt) assay.

For each in vitro experiment, two controls were set up in identical conditions including an $\mathrm{IC}_{0}\left(0 \%\right.$ inhibitory concentration; cells only) and an $\mathrm{IC}_{100}(100 \%$ inhibitory concentration; media only), and exposed to air only during the exposure time.

\subsection{Toxicity determination of individual chemicals and their mixtures}

Test chemicals were freshly prepared each time, immediately before use. Range finding experiments were undertaken to determine the concentration range of individual test chemicals that inhibited the viability of $50 \%$ exposed cells. In general for any binary, ternary, quinary mixtures of selected toxicants, the previously experimentally derived $\mathrm{IC}_{50}$ of each toxicant in the mixture was used as an index of toxicity. The toxicants were prepared such that their fractional effects individually in the mixture was calculated in proportion of their concentration to the total concentrations of all toxicants in the mixture and such that the sum of all ratio combinations equalled to a theoretical additive value of 1. E.g. for chemicals A, B and C;

$$
\frac{I C_{50}(A)}{I C_{50}(\text { mix })}+\frac{I C_{50}(B)}{I C_{50}(\text { mix })}+\frac{I C_{50}(C)}{I C_{50}(\text { mix })}=1
$$

$\mathrm{IC}_{50}(\mathrm{~A}, \mathrm{~B}$ or $\mathrm{C}$ ) denotes $50 \%$ inhibitory concentration for chemical $\mathrm{A}, \mathrm{B}$ or $\mathrm{C}$ when administered individually and calculated with the respective in vitro tests.

$\mathrm{IC}_{50 \text { (тіх) }}$ is the sum of the total individual $\mathrm{IC}_{50}$ concentrations for the chemicals. For chemicals A, B and C, for e.g. the mixture ratio of chemical

$$
\mathrm{A}=1-\left(\mathrm{IC}_{50 \mathrm{~B}} / \mathrm{IC}_{50 \text { mix }}-\mathrm{IC}_{50 \mathrm{C}} / \mathrm{IC}_{50 \text { mix }}\right)
$$

and so forth the remaining chemicals. After having the $\mathrm{IC}_{50}$ values of individual chemicals, ratios of mixture components were calculated using the equation above and then $\mathrm{IC}_{50}$ values were determined for binary, ternary and quinary mixtures as per procedure described above and dose response curves were generated accordingly (Fig $1 \& 2$ ). Once $\mathrm{IC}_{50}$ values of individual and chemical 
mixtures were calculated, interaction effect was estimated using isobole method briefly discussed in a later section.

\subsection{Cytotoxicity assays}

The MTS assay (Promega, USA) was selected for measuring the number of active cells in the culture (based on the lactate dehydrogenase activity in the mitochondria). The MTS assay measuring the conversion of a soluble tetrazolium salt to a formazan product by viable cells [9]. The assay consisted of an MTS solution prepared by mixing a solution of MTS (42 mg MTS powder in $21 \mathrm{ml}$ of DPBS $\mathrm{pH} 6.0-6.5)$ with a PMS solution $(0.92 \mathrm{mg} / \mathrm{ml}$ PMS in DPBS) to the cells to be tested in a ratio of 1:5. The MTS was then incubated with the cells for a period of $2 \mathrm{~h}$ at $37^{\circ} \mathrm{C}$ in the dark. After $2 \mathrm{~h}$, the cellular supernatant absorbance was measured. The amount of reduced Formazan was assessed by measuring the optical density at $492 \mathrm{~nm}$ using a Labsystem Multiskan MS plate reader. Data was plotted as a dose response curve exposure versus absorbance reading

\subsection{Statistical analysis}

Dose response curves reported were plotted from experimental data. All data reported was expressed as mean \pm SD of 3-4 replicated wells. Statistical procedures and graphical analysis were performed using Graphpad Prism software.

\subsection{Results}

The dose response curves for individual VOCs and their mixtures were generated using the static methodology as outlined in Figures $1 \& 2$. The graphs are mainly presenting the experimental data from a series of experiments using airborne concentrations of individual and mixtures of generated VOCs.

Once $\mathrm{IC}_{50}$ values of individual and chemical mixtures were calculated, interaction effect was estimated by using isobole method [10]. The isobole

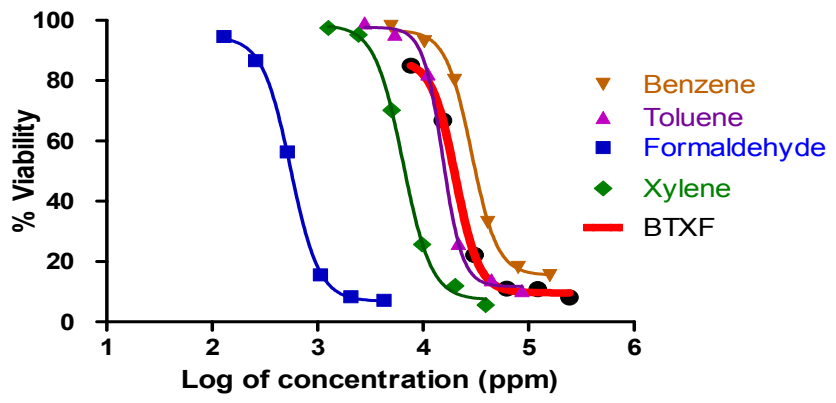

Figure 1: $\quad$ Individual and mixtures cytotoxicity in lung cells (A549). 


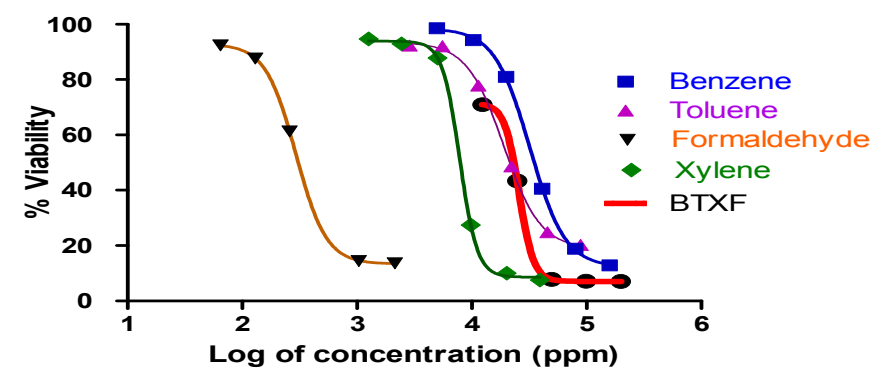

Figure 2: $\quad$ Individual and mixtures cytotoxicity in liver cells (HepG2).

method is based on the assumption that if A and B are applied jointly their mixture toxicity can be estimated by dividing the concentration of each toxicant in the mixture with the concentration of the toxicants applied singly that yields the same effect as the mixture. The method is valid for mixtures of any given number of toxicants [11]. Mathematically, isobole method for an additive mixture effect can be described as:

$$
\frac{d A}{D A}+\frac{d B}{D B}=1
$$

$\mathrm{dA}$ and $\mathrm{dB}=$ the dose of chemical $\mathrm{A}$ and $\mathrm{B}$ in the mixture which produces a given effect while $\mathrm{DA}$ and $\mathrm{DB}=$ the dose of chemical $\mathrm{A}$ and $\mathrm{B}$ in single toxicant experiments which elicits the same effect as the mixture.

If the isobole calculation yields a figure less than 1 then the relationship is synergistic. Furthermore if the calculation is more than 1 then the relationship can be classified as antagonistic (Table 2).

Table 1: $\quad$ Chemical toxicity parameters.

\begin{tabular}{|c|c|c|}
\hline Chemicals & $\begin{array}{c}\text { Lung Cells } \\
\text { (A549) } \\
\text { IC }_{50}(\mathrm{ppm})\end{array}$ & $\begin{array}{c}\text { Liver Cells } \\
\text { (HepG2) } \mathrm{IC}_{50}(\mathrm{ppm})\end{array}$ \\
\hline Benzene & $29915 \pm 1103$ & $33113 \pm 1250$ \\
\hline Toluene & $14217 \pm 1132$ & $17721 \pm 126$ \\
\hline Xylene & $6847 \pm 792$ & $7453 \pm 830$ \\
\hline Formaldehyde & $524 \pm 105$ & $305 \pm 84$ \\
\hline Benzene: Toluene & $29509 \pm 563$ & $4634 \pm 1198$ \\
\hline Benzene:Xylene & $29006 \pm 849$ & $28183 \pm 1102$ \\
\hline Toluene: Xylene & $18465 \pm 746$ & $20550 \pm 716$ \\
\hline Benzene: Formaldehyde & $17715 \pm 208$ & $14043 \pm 388$ \\
\hline Benzene:Toluene:Xylene & $34062 \pm 2626$ & $33424 \pm 1988$ \\
\hline Benz:Tol:Xyl:Form & $21999 \pm 1775$ & $28739 \pm 2957$ \\
\hline
\end{tabular}


Table 2: $\quad$ Interactive effects of VOC mixtures.

\begin{tabular}{|c|c|c|c|c|}
\hline & \multicolumn{2}{|c|}{ A 549 Cells } & \multicolumn{2}{c|}{ Hep G2 Cells } \\
\hline Chemical Interaction & Ratio & $\begin{array}{c}\text { Interaction } \\
\text { Effect }\end{array}$ & Ratio & $\begin{array}{c}\text { Interaction } \\
\text { Effect }\end{array}$ \\
\hline Benz:Toluene & $1.31-1.35$ & Antagonistic & $1.71-1.79$ & Antagonistic \\
\hline Benz:Xylene & $1.56-1.58$ & Antagonistic & $1.32-1.48$ & Antagonistic \\
\hline Toluene:Xylene & $1.74-1.76$ & Antagonistic & $1.60-1.68$ & Antagonistic \\
\hline Benz:Formaldehyde & $0.90-0.92$ & Synergistic & $0.82-0.92$ & Synergistic \\
\hline Benz:Tol:Xylene & $1.85-2.16$ & Antagonistic & $1.61-1.86$ & Antagonistic \\
\hline $\begin{array}{c}\text { Benz:Tol:Xylene: } \\
\text { Formaldehyde }\end{array}$ & $1.54-1.80$ & Antagonistic & $2.2-2.5$ & Antagonistic \\
\hline
\end{tabular}

\section{Discussion}

There are a large number of known chemicals in the natural and built environment that humans are exposed to in addition to an ever increasing number of new chemicals and mixtures for which no data exists [12]. The lungs, skin, central nervous system (CNS), liver and the kidneys are the main body systems affected by these chemicals [13]. Testing, even for the most potent mixtures with classical toxicological protocols, is unrealistic and perhaps not achievable. Most federal agencies and international organisations such as ATSDR, US EPA, NIOSH use a default assumption of response additivity in assessing mixture toxicity in exposed human populations [10, 14]. However this assumption has setbacks as it does not factor chemical interactions in toxicity determination. There is also a lack in direct comparison between the available approaches for mixtures toxicity assessment, as most tend to differ in data types and nature of observations as reported in the literature [15]. No published in vitro airborne toxicity data could be sourced for mixtures of benzene, xylene, toluene and formaldehyde. However, inhalational in vivo toxicity data for Toluene and Xylene have been reported in rat by the NIOSH Registry of Toxic Effects of Chemical Substances (RTECS).

Figures 1 and 2 indicated the toxicity of individual VOCs and their mixtures. The striking feature in the two graphs is the non additive effect of BTXF mixtures (when compared to individual VOC curves) which suggested a non additive effect resulting from ternary mixtures. The results of individual chemicals (Table 1) reported the cytotoxicity of formaldehyde in liver cells $\left(\mathrm{IC}_{50}=305+84 \mathrm{ppm}\right)$ was quite higher than that in lung cells $\left(\mathrm{IC}_{50}\right.$ $=524+105 \mathrm{ppm})$. Among structurally similar VOC's, the cytotoxicity of xylene $\left(\mathrm{IC}_{50}=6847 \pm 792 \mathrm{ppm}\right.$, A549 cells; $7453 \pm 830$ ppm, Hep-G2) was found 2-3 times higher than that of toluene $\left(\mathrm{IC}_{50}=14216 \pm 132 \mathrm{ppm}, \mathrm{A} 549\right.$; $17721 \pm 1226 \mathrm{ppm}, \mathrm{HepG} 2)$ and five times higher than benzene $\left(\mathrm{IC}_{50}=\right.$ 29915 \pm 1103 ppm, A549; $33113 \pm 1250$ ppm, HepG2). Among four selected chemicals, low weight carbonyl compound, formaldehyde was more toxic while cytotoxic effect of well-recognised carcinogenic compound i.e. benzene was less than other chemicals. The $\mathrm{LC}_{50}(50 \%$ Lethal Concentration) 
values of xylene $(5000 \mathrm{ppm})$ and toluene $(13,000 \mathrm{ppm})$ have been reported in rats following $4 \mathrm{~h}$ exposure $[16,17]$. Based on in vitro results, $\mathrm{IC}_{50}$ values for xylene (6847 $\pm 792 \mathrm{ppm})$ and toluene $(14217 \pm 1132 \mathrm{ppm})$ for human lung cells were determined after $1 \mathrm{~h}$ exposure (Table 1$)$. An in vitro/in vivo comparison indicates that the in vitro toxicity findings in the present study are in good correlation with inhalational in vivo published data for both volatile organic solvents. The $\mathrm{LC}_{50}$ for benzene $(10,000 \mathrm{ppm})$ have been reported in rats following $7 \mathrm{~h}$ exposure [18]. The observed in vitro $\mathrm{IC}_{50}$ values for benzene (1 $\mathrm{h}$ exposure) are $29915 \pm 1103$ ppm (A549 cells) and $33113 \pm 1250$ ppm (HepG2 cells) which clearly indicates the higher sensitivity achieved by using the developed in vitro methodology. Regarding formaldehyde, the lethal concentration $\left(\mathrm{LC}_{50}\right)$ for inhalation $\mathrm{s}$ reported in the literature ranged from $405 \mathrm{ppm}\left(497 \mathrm{mg} / \mathrm{m}^{3}\right)$ in mice to $471 \mathrm{ppm}\left(578 \mathrm{mg} / \mathrm{m}^{3}\right)$ in rats following four hours exposure [19]. In comparison, in this study $\mathrm{IC}_{50}$ values for in vitro toxicity of formaldehyde in A549 cells and HepG2 cells were found to be $524 \pm 105 \mathrm{ppm}$ and $305 \pm 84 \mathrm{ppm}$ respectively. In vivo published data suggest that the cytotoxic effects of formaldehyde in laboratory animals appears to be more closely related to the exposure level of formaldehyde than to the time of exposure or total dose $[20,21]$.

Binary and ternary mixtures of benzene, toluene and xylene showed antagonistic effects on human lung and liver cells. A study by Ewa and Anna (2008), on binary effect of toluene and xylene on lipid peroxidation also reported an antagonistic effect [22]. Briefly, the binary mixtures of benzene: formaldehyde exerts synergistic effects in human lung and liver cells while binary and ternary mixtures of structurally similar VOCs i.e. Benzene: Toluene: Xylene demonstrated antagonistic effects on both types of human cells (Table 2). Toxicity of quinary mixtures of BTXF was higher (A549 IC $_{50}$ : 21999 \pm 1775 ; HepG2 $\mathrm{IC}_{50}: 28739 \pm 2957$ ) when compared to ternary mixtures of BTX (A549 $\mathrm{IC}_{50}$ : $34062 \pm 2626$; HepG2 $\mathrm{IC}_{50}: 33424 \pm 1988$ ). This may be due to presence of formaldehyde in quinary mixture however combined effect of BTX was dominant over formaldehyde toxicity hence overall effect of BTXF quinary mixture was antagonistic.

It is concluded from the data presented there are possible toxicological interactions (i.e. departures from additivity) that have clear implications for risk assessment. The presented data clearly highlighted the limitations of an additive interaction assumption and the need to focus on volatile chemical mixture studies. This is an important factor to consider because real life exposures consist of exposure to a cocktail of numerous chemicals rather than single individual chemical. The in vitro cytoxicity studies conducted are relevant and important to risk assessment of chemical mixtures in several ways. The results showed that chemicals in a mixture do not necessarily act in an additive fashion and the possible inclusion of cytotoxicity assays can help in the regulatory decision making process. The study may have implications for risk assessment of environmental exposure and establishing safe levels of exposure. Studies are being conducted looking at a dynamic exposure to these VOC mixtures and their immunotoxic/genotoxic effect on human health. 


\section{Acknowledgement}

The project was funded by the Faculty of Science research grant initiative, UNSW.

\section{References}

[1] Boekelheide, K. (2007). Mixed messages. Toxicol. Sci. 99, 1-2.

[2] Feron, V. J., and Groten, J. P. (2002). Toxicological evaluation of chemical mixtures. Food Chem. Toxicol. 40, 825-839.

[3] ANZECC. Australian and New Zealand guidelines for the assessment and management of contaminated sites. Australian and New Zealand Environment and Conservation Council / National Health and Medical Council, 1992.

[4] Azzi, R., Hayes, A., Khalil, C., \& Winder, C. An in vitro study of the interactive effect of 24 binary and ternary mixtures from the GHS classification groups. ALTEX 22 (Spl):128, 2005.

[5] Belden, J.B., Gilliom, R.J., Martin, J.D., Lydy, M.J. Relative toxicity and occurrence patterns of pesticide mixtures in streams draining agricultural watersheds dominated by corn and soybean production. Integrated Environmental Assessment \& Management. 3(1):90-100, 2007.

[6] Khalil, C. Combining Three in vitro assays for detecting early signs of UVB cytotoxicity in cultured human skin fibroblasts. WIT Transactions on Biomedicine and Health (10): 349-359, 2006.

[7] Bakand, S., Winder, C., Khalil, C., Hayes, A novel in vitro exposure technique for toxicity testing of selected volatile organic compounds. J. Enviro. Monitoring. (8): 100-105.

[8] Salvatore R. DiNardi, 2003, Occupational Environment: Its Evaluation, Control, And Management, 2nd Edition, ISBN-13: 978-1-931504-43-1

[9] Promega. CellTiter $96^{\circledR}$ Aqueous Non-Radioactive Cell Proliferation Assay: Technical Bulletin No 169. Promega Corporation. Madison, USA.

[10] ATSDR (2004). Guidance manual for the assessment of joint toxic action of chemical mixtures. Agency for Toxic Substances and Disease Registry. US Department of Health and Human Services, Atlanta].

[11] Poch, G., R. J. Reiffenstein and H. D. Unkelbach (1990). "Application of the Isobologram Technique for the Analysis of Combined Effects with Respect to Additivity as Well as Independence." Canadian Journal of Physiology and Pharmacology 68. pp 682-688

[12] Marinovich, M., Ghilardi F. and Galli C. L. (1996). "Effect of Pesticide Mixtures on in Vitro Nervous Cells: Comparison with Single Pesticides." Toxicology 108: 201-206.

[13] Winder, C., 2004a. Occupational respiratory diseases. In: Occupational Toxicology, Second edition. Winder, C. and Stacey, N. H. (Eds). CRC Press, Boca Raton, pp. 71-114.

[14] U.S. EPA. (2000) Supplementary guidance for conducting health risk assessment of chemical mixtures. EPA/630/R-00/002 
[15] Pounds, K. G., Haider J., Chen D. G. and Mumtaz M. (2004). "Interactive Toxicity of Simple Chemical Mixtures of Cadmium, Mercury, Methylmercury and Trimethyltin: Model-Dependent Responses." Environmental Toxicology and Pharmacology. 18: 101- 113.

[16] NIOSH, The Registry of Toxic Effects of Chemical Substances, Toluene, RTECS \# XS5250000, 2004.

[17] NIOSH, 2004b, The Registry of Toxic Effects of Chemical Substances, Xylene, RTECS \# ZE2100000, 2004.

[18] ATSDR (2007), Toxicological Profile for Benzene. U.S. Department of Health and Human Services: Agency for Toxic Substances and Disease Registry, Atlanta, GA.

[19] IPCS, 1989. Formaldehyde. Environmental Health Criteria No 89. International Programme on Chemical Safety. World Health Organization, Geneva.

[20] IARC, 1995. International Agency for Research on cancer- Summaries \& Evaluations, Formaldehyde, Vol 62.

[21] Wilmer, J. W., Woutersen, R. A., Appelman, L. M., Leeman, W. R. and Feron, V. J., 1989. Subchronic (13-week) inhalation toxicity study of formaldehyde in male rats: 8-hour intermittent versus 8-hour continuous exposure. Toxicology Letters, 47:3, 287-293.

[22] Ewa Sawicka and Anna Długosz, Toluene And P-Xylene Mixture Exerts Antagonistic Effect On Lipid Peroxidation In Vitro, International Journal of Occupational Medicine and Environmental Health 2008; 21(3):201-209. 